\title{
Returning to Surgery-Experience, Discussions and Consensus
}

\author{
Matthew Kroh ${ }^{1}$ - Gerhard Prager ${ }^{2}$ • Francesco Rubino ${ }^{3}$ - Giuseppe Maria Marinari ${ }^{4}$. Antonio J. Torres ${ }^{5}$. \\ Shaw Somers ${ }^{6} \cdot$ Fakı Akın ${ }^{7}$. Owen Haskins ${ }^{8}$ (i)
}

Received: 10 August 2020 / Revised: 11 September 2020 / Accepted: 15 September 2020 / Published online: 23 September 2020

(C) Springer Science+Business Media, LLC, part of Springer Nature 2020

\section{Dear Editor,}

Severe acute respiratory syndrome coronavirus 2 and its related disease, coronavirus disease 2019 (COVID-19) that emerged from Wuhan, China, in December 2019, can cause severe disease, pneumonia, severe acute respiratory syndrome, kidney failure, and death [1-4]. On 11 March 2020, the World Health Organization declared the virus a pandemic [5].

In response, global healthcare systems postponed most elective surgeries [6-8], including bariatric and metabolic (B\&M) surgery, to mitigate the spread of the virus, allow the reallocation of resources to those patients most in need [6] and prevent health systems from becoming overwhelmed.

The incidence of COVID-19 has subsided in some regions, and subsequently, some elective B\&M surgery has resumed $[9,10]$. B\&M societies have called for the appropriate safe restart of surgery $[11,12]$, and guidelines and

\section{Owen Haskins}

1 Digestive Disease Institute, Cleveland Clinic Abu Dhabi, Cleveland Clinic Lerner College of Medicine, PO Box: 112412, Abu Dhabi, United Arab Emirates

2 Department of Surgery, Medical University of Vienna, AKH Wien Leitstelle 21A, Waehringer Guertel 18-20, A-1090 Wien, Austria

3 King's College Hospital NHS Foundation Trust, Denmark Hill, Brixton, London SE5 9RS, UK

4 Humanitas Research Hospital, IRCCS, Via Alessandro Manzoni, 56, 20089 Rozzano, MI, Italy

5 Department of Surgery, Hospital Clínico San Carlos, Complutense University of Madrid, Calle del Prof Martín Lagos, s/n, 28040 Madrid, Spain

6 Streamline Clinics Ltd UK, The Sudbury Clinic, Clementine Churchill Hospital, Sudbury Hill, Harrow HA1 3RX, UK

7 Bariatric \& Metabolic Surgery, Istanbul, Turkey

8 Biran, France recommendations for the safe resumption of B\&M surgery have been published by societies [9, 13-17]. Nevertheless, doubts remain about when and how $\mathrm{B} \& \mathrm{M}$ centres will resume surgery.

\section{COVID-19 Roundtable}

On 24th June 2020, a 'COVID-19 Roundtable' consensus meeting was convened comprised of a panel of B\&M surgery experts who shared their experiences and discussed the criteria that their centres/countries are deploying to safely return to surgery and developed consensus statements.

Moderated by Professor Gerhard Prager (Medical University of Vienna, Austria), the Roundtable began with a presentation by Dr. Matthew Kroh (Chair of the Digestive Disease Institute at Cleveland Clinic Abu Dhabi, UAE) who highlighted how COVID-19 has impacted his practise and organisation. Elective procedures were stopped in Abu Dhabi in mid-March, with only emergency general surgery performed and ICU beds reserved for the most critical cases. In the operating room (OR), the numbers of personnel and equipment were reduced, and the use of personal protective equipment (PPE) on wards increased when managing known COVID patients. Where possible, the enhanced recovery after surgery (ERAS) programme was accelerated to help reduce the length of hospital stay.

Professor Francesco Rubino (Kings College London, London, UK) said unlike other surgical specialities, B\&M surgery has never conceived a prioritisation system that recognises urgency of surgical treatment based on need. Traditional 'eligibility criteria' in BMS do not reflect disease burden, and priority access to surgery is often decided on a 'first come, first serve' basis. Coupled with stigma, weight bias and the misconception of B\&M surgery as 'a last resort', the absence of an objective measure of urgency undermines access to B\&M surgery. 
Obesity and the COVID pandemic clearly dispelled the misconception that B\&M surgery is 'cosmetic', it is lifesaving. B\&M patients have differing burden of disease, and a significant amount of harm (depending on the type and severity of their comorbidities) can be done to some patients by delaying treatment.

A prioritisation system based on burden of disease, as outlined by the Diabetes Surgery Summit (DSS) [14], would prioritise patients on the basis of the diseases/disease stages that are most likely to progress if treatment is delayed.

Professor Antonio Torres (Hospital Clínico San Carlos, Madrid, Spain) said that the pandemic decimated bariatric services across Spain with specialists reallocated to different healthcare roles. To manage the outbreak, the Hospital Clínico San Carlos was divided into 2 areas - 'COVID free' and 'COVID area'. Patients with no symptoms who tested negative were admitted on the day of the operation and admitted straight to the OR, and ERAS protocols were implemented, with patients staying a maximum of 2 days in hospital.

B\&M was resumed the first week of June, and follow-up was performed via telemedicine. However, volume remains exceptionally low primarily through patient fear of contracting COVID in hospitals. Approximately $70 \%$ of bariatric surgeons in Spain do not know when they will resume surgery.

In Turkey, Dr. Faki Akin (Istanbul, Turkey) explained that most private hospitals are 'non-pandemic' and most state/ public hospitals are 'pandemic hospitals'. Elective surgery was stopped on 17 March and began again on 1 July in public hospitals.

A survey of Turkish bariatric surgeons carried out at the end of May revealed that:

- No surgeons tested for COVID post-operatively.

- $60 \%$ of surgeons thought there would be a second wave.

- A majority said they would perform PCR and tomography testing, would treat patients from abroad and would use telemedicine.

In the UK, Mr. Shaw Somers (Portsmouth Hospitals NHS Trust and Streamline Surgical, UK) revealed that all elective surgery was postponed in mid-March, and all doctors effectively became COVID doctors. All the private hospitals in the UK were commandeered by the government to treat nonCOVID emergencies and cancer patients, and the only bariatric procedures performed were emergencies (slipped bands, ulcers, etc.).

Currently, B\&M surgeons are fearful that surgery on the NHS may not resume for a long time due to the misconception that it is a cosmetic procedure. Therefore, it is important that $\mathrm{B} \& \mathrm{M}$ centres demonstrate to governments that if patients are screened properly and appropriate pathways implemented, B\&M surgery can be performed safely.
In Italy, elective B\&M surgery was stopped on 22 February and resumed on 20 May; initially it was only at $60 \%$ of preCOVID levels to ensure that ICU capacity was maintained, Dr. Giuseppe Marinari (Humanitas Research Hospital, Milan, Italy) reported. The Humanitas Research Hospital has followed the Diabetes Surgery Summit (DSS) recommendations [14] of operating on the most complex cases, excluding patients aged $>60$, BMI $>60$ and challenging revision cases [17]. Surgical protocols are localised depending on the local situation, ERAS has been adopted, and patients are only sent to ICU in the event of complications.

\section{Roundtable Discussions}

In the roundtable discussions, it was reiterated that with the right screening and pathways, bariatric surgery should be resumed immediately, and B\&M surgeons must explain the severity of obesity as an illness and that the lack of surgery will increase obesity-related deaths. B\&M surgeons must also have a clear message about the benefits of surgery, its positive impact on life-threatening co-morbidities and improvement in patients' quality of health.

Many B\&M candidates are affected by severe illnesses that carry a risk of life-threatening complications if left untreated; the theoretical risk of severe COVID-19 should be considered in the context of the need to urgently treat a life-threatening disease.

\section{Consensus Outcomes Statements}

- B\&M surgery is the most effective and durable treatment for people living with severe obesity, and the impact of obesity and diabetes on COVID19 severity demonstrates why surgery should be immediately restarted with the number of procedures increased.

- A greater emphasis should be placed on patient-focused prioritisation system considering the diseases that are most likely to be improved post-operatively and progress/ deteriorate if left untreated

- Necessary elements of the ERAS protocol can be adopted to reduce length of hospital stay, OR personnel and equipment reduced and staff educated to manage suspected COVID-19 patients.

- B\&M specialists must develop a coherent message raising awareness about the benefits of surgery.

- Telemedicine embraced by patients and B\&M specialists could dramatically change follow-up regimes and increase patient-provider contact in novel ways.

- Social media should be embraced by B\&M surgeons to promote the benefits of surgery and improve communication with patients. 


\section{Conclusion}

The decision to restart B\&M surgery should be measured and judicious, and the potential risks to and safety of patients and staff are paramount. A patient-focused prioritisation system, considering the diseases and conditions that are most likely to be improved post-operatively, should be implemented.

Obesity and diabetes are major risk factors for hospitalisation and mortality due to COVID-19, and considering $\mathrm{B} \& \mathrm{M}$ surgery is the most effective treatment to aid the resolution of both these debilitating conditions, delaying and/ or denying surgery is depriving these patients of a safe, effective and life-changing procedure.

B\&M surgeons need to re-energise their efforts to promote the many benefits of surgery and engage with all stakeholders to vigorously challenge weight stigma and bias. Due to the impact of the pandemic, it is a unique opportunity for $\mathrm{B} \& \mathrm{M}$ surgeons to at last change the 'cosmetic surgery' perception to 'life-saving surgery'. If not now, then when?

Funding The series of Expert Roundtables was funded by Medtronic Inc.

\section{Compliance with Ethical Standards}

Conflict of Interest Gerhard Prager has no conflict of interest to report. Matthew Kroh has no conflict of interest to report.

Francesco Rubino has no conflict of interest to report.

Antonio Torres has no conflict of interest to report.

Faki Akin has no conflict of interest to report.

Shaw Somers has no conflict of interest to report.

Giuseppe Marinari has no conflict of interest to report.

Owen Haskins received payment from Medtronic for writing the paper.

Ethical Approval Study did not require approval from the ethics committee.

Human and Animal Rights This article does not contain any studies with human participants or animals performed by any of the authors.

Informed Consent Informed consent does not apply.

\section{References}

1. World Health Organization. Clinical management of severe acute respiratory infection when novel coronavirus $(\mathrm{nCoV})$ infection is suspected: interim guidance, 25 January 2020. World Health Organization, 2020.

2. Wang $\mathrm{D}, \mathrm{Hu} \mathrm{B}, \mathrm{Hu} \mathrm{C}$, et al. Clinical characteristics of 138 hospitalized patients with 2019 novel coronavirus-infected pneumonia in Wuhan, China. JAMA. 2020;323(11):1061-9.

3. Spinelli A, Pellino G. COVID-19 pandemic: perspectives on an unfolding crisis. Br J Surg 2020;10

4. Lai CC, Shih TP, Ko WC, et al. Severe acute respiratory syndrome coronavirus 2 (SARS-CoV-2) and corona virus disease-2019
(COVID-19): the epidemic and the challenges. Int J Antimicrob Agents 2020;105924

5. WHO Director-General's opening remarks at the media briefing on COVID-19: https://www.who.int/dg/speeches/detail/who-directorgeneral-s-opening-remarks-at-the-media-briefing-on-covid-19\% 2D\%2D-11-march-2020

6. CMS Releases Recommendations on Adult Elective Surgeries, Non-Essential Medical, Surgical, and Dental Procedures During COVID-19 Response - https://www.cms.gov/newsroom/pressreleases/cms-releases-recommendations-adult-elective-surgeriesnon-essential-medical-surgical-and-dental

7. NHS England and NHS Improvement. Letter to chief executives of all NHS trusts and foundation trusts, CCG accountable officers, GP practices and primary care networks, and providers of community health services. 17 Mar 2020. https://www.england.nhs.uk/ coronavirus/wp-content/uploads/sites/52/2020/03/urgent-nextsteps-on-nhs-response-to-covid-19-letter-simon-stevens.pdf

8. Australian Health Protection Principal Committee (AHPPC) advice to National Cabinet on 24 March 2020 - https://www.health.gov.au/ news/australian-health-protection-principal-committee-ahppcadvice-to-national-cabinet-on-24-march-2020

9. Dong Z, Zhang P, Zhu J, et al. Recommendations to manage patients for bariatric surgery in the COVID-19 pandemic: experience from China. Obes Surg. 2020; https://doi.org/10.1007/s11695-02004741-8.

10. Uccelli M, Cesana GC, Ciccarese F, et al. COVID-19 and obesity: postoperative risk in patients who have undergone bariatric surgery. Preliminary Report from High Volume Center in Italy (Lombardy). Obes Surg. 2020; https://doi.org/10.1007/s11695-020-04792.

11. Executive Council of ASMBS - Safer through surgery: American Society for Metabolic and Bariatric Surgery statement regarding metabolic and bariatric surgery during the COVID-19 pandemic (Surg Obes Relat Dis (2020) 1-2; https://doi.org/10.1016/j.soard. 2020.06.003)

12. BOMSS Council letter to PM 19 May $2020 \mathrm{https} / / / \mathrm{www} \cdot b o m s s$. org.uk/wp-content/uploads/2020/05/BOMSS-Council-Letter-toPM-19-May-2020.pdf

13. Khidir $\mathrm{N}$ et al. How are we going to restart elective bariatric and metabolic surgery after the peak of COVID-19 pandemic? Sur Res Rep. 2020;3(1):2-5. https://www.ifso.com/pdf/restart-electivebariatric-and-metabolic-surgery-after-the-peak-of-covid-19pandemic.pdf

14. Rubino et al. Bariatric and metabolic surgery during and after the COVID-19 pandemic: DSS recommendations for management of surgical candidates and postoperative patients and prioritisation of access to surgery. Lancet Diabetes Endocrinol. 2020; https://doi. org/10.1016/S2213-8587(20)30157-1.

15. Reintroducing Safe Bariatric Surgery - BOMSS Guidance 28th May 2020 https://www.bomss.org.uk/wp-content/uploads/2020/ 06/BOMSS-Restarting-Bariatric-Surgery-in-the-COVID-EraGuidelines_May-2020.pdf

16. Navarra G, Komaei I, Currò G, et al. Bariatric surgery and the COVID-19 pandemic: SICOB recommendations on how to perform surgery during the outbreak and when to resume the activities in phase 2 of lockdown. Updat Surg. 2020;72:259-68. https://doi. org/10.1007/s13304-020-00821-7.

17. Marinari GM, Anselmino M, Tascini C, et al. Bariatric and metabolic surgery during COVID-19 outbreak phase-2 in Italy: why, when and how to restart. SOARD. 2020; https://doi.org/10.1016/j. soard.2020.06.025.

Publisher's Note Springer Nature remains neutral with regard to jurisdictional claims in published maps and institutional affiliations. 